\title{
Identification of a mutant from Arachis veigae with enhanced seed oleic and very long-chain fatty acid content
}

\author{
Brandon Tonnis ${ }^{1}$, Ming Li Wang ${ }^{1 *} \mathbb{D}$, Shyam Tallury', Viktor Tishchenko ${ }^{2}$ and H. Thomas Stalker ${ }^{3}$
}

\begin{abstract}
High oleate is an important seed quality trait frequently incorporated in peanut varieties. Crop wild relatives (CWR) are potentially useful genetic resources for cultivar improvement through genetic introgression; but for wild peanut species, many chemical or nutritional traits are not well characterized. A mutant from Arachis veigae S. H. Santana \& Valls $(2 n=2 x=20)$, with increased oleic and very long chain $(C \geq 22)$ fatty acid content was identified from screening 209 accessions of 45 species using gas chromatography (GC). The A. veigae (formerly A. sylvestris) accession, WVeSv 8373 (PI 688970) contained 55.5\% oleic acid in seeds, significantly higher than the average (18.3\%) of other accessions within the same species and also significantly higher than the average (37.0\%) of all wild peanut accessions evaluated. A C37T substitution was identified by sequencing the coding region of $F A D 2 H$, resulting in the nonsense mutation of Q13* (a premature stop codon). This functional mutation may significantly reduce the fatty acid desaturase (FAD) activity and result in the enhanced oleate level. Arachis veigae also contained a high percentage of very long-chain $(C \geq 22)$ fatty acids, and their variation identified in this study is also discussed and compared with other species. The mutant with such an altered fatty acid composition may be useful for potentially improving seed or food nutrition quality.
\end{abstract}

Keywords: Wild peanut species, FAD2 coding region, Natural point mutation, Fatty acid composition, Nutrition quality

\section{Introduction}

Peanut (Arachis hypogaea L.) is an important oilseed crop which is grown worldwide and mainly used for oil production. It also contains a high percentage of protein $(\sim 25 \%)$ making it an important source of nutrition, especially for many in underdeveloped countries. In addition to oil and protein, many other useful compounds (polyphenols, antioxidants, minerals, vitamins, and resveratrol) have been identified in peanuts. Consuming food products with these compounds can benefit human health. Therefore, peanut can be considered a functional food [1]. Crop wild relatives (CWR) are valuable germplasm resources for new cultivar development

\footnotetext{
*Correspondence: MingLi.Wang@ars.usda.gov

1 USDA-ARS, Plant Genetic Resources Conservation Unit, University

of Georgia, 1109 Experiment Street, Griffin, GA 30223, USA

Full list of author information is available at the end of the article
}

and improvement [2]. There are 82 species including the cultivated peanut $A$. hypogaea within the genus Arachis [3-5], and except $A$. hypogaea, the remaining are considered as peanut wild relatives. Some of the wild species have been successfully used for peanut cultivar improvement, especially for disease and insect resistance [6-12]. More detailed information can be found in a recent review by Stalker [13]. However, research on seed quality of wild peanut species has been minimal, and only a limited number [75, 17 and 39] of wild species were evaluated for certain quality traits including protein and oil content, fatty acid composition, and sterol composition [14-16], respectively. Additional information on seed quality traits will be valuable for using wild species for nutritional enhancement of cultivated peanut.

The Plant Genetic Resources Conservation Unit (PGRCU) of USDA-ARS in Griffin, GA, maintains a large number of wild peanut species accessions. To evaluate 
the seed quality of wild peanut species, 209 accessions covering 45 species were grown in a greenhouse; and freshly harvested seeds were used for chemical analysis including oil content, protein content, and fatty acid composition as well as relative seed size [17]. From this screening, one mutant in A. veigae was identified with a higher level of oleic acid. Arachis veigae was previously classified as $A$. sylvestris, which is a highly dispersed wild species within the genus [5]. It is a diploid species with an $\mathrm{H}$ genome [13] and belongs to the section Heteranthae. It is significantly diverged from the A and B genomes of diploid species of section Arachis, the section that includes the cultivated peanut. Therefore, the objectives of this study were to (1) determine the fatty acid profile of this mutant line, (2) identify the functional mutation on the $F A D 2 H$ gene by sequence analysis, and (3) compare fatty acid profiles of accessions of $A$. veigae and selected diploid wild species.

\section{Materials and methods Collection of peanut seeds}

Seeds of 209 accessions of 45 wild peanut species were obtained from the PGRCU, GA. In 2016, five seeds from each accession were planted in $14^{\prime \prime}$ diameter panterra pots. After maturation, the pods were harvested from each plant, and four accessions (PI 468202, A. duranensis representing genome A; PI 468184, A. valida representing genome B; PI 688970, and PI 688983, A. veigae representing genome $\mathrm{H}$ ) were selected for this study. Freshly harvested seeds were used for chemical analysis.

\section{Chemical analysis of fatty acid composition using gas chromatography}

Fatty acid analysis followed a previously published method by our laboratory [18]. Ten to 20 seeds from each accession were ground to a powder, and a small amount was sampled for preparing fatty acid methyl esters by alkaline transmethylation. Fatty acid composition was determined by gas chromatography for identifying and calculating relative peak areas. Measurements for each sample were performed in duplicate.

\section{Sequence analysis and comparison of FAD2 coding region}

Freshly-harvested seeds from the four accessions used for chemical analysis plus two cultivated materials (Tifrunner and F435) were grown in the greenhouse for DNA extraction. Leaf tissue (75-100 mg) was collected from freshly unfolded young leaves, and DNA was extracted using an Omega Bio-Tek E.Z.N.A. Plant DNA kit (Norcross, GA). DNA quality and quantity were determined on a Nanodrop 2000C spectrophotometer, and DNA concentration was adjusted to $10 \mathrm{ng} / \mu \mathrm{l}$ as template for amplifying the FAD2 coding region. Initially, gene-specific primers for FAD2A (5' GAT TAT TGA CTT GCT TTG TAG TAG TGC $3^{\prime}$ and $5^{\prime}$ ACA CAA ACG TTT TCA ACT CTG AC $\left.3^{\prime}\right)$ and FAD2B (5' CAG AAC CAT TAG CTT TGT AGT AGT GC $3^{\prime}$ and $5^{\prime}$ ACA CAA ACG TTT CCA ACT CTG AC $3^{\prime}$ ) were tested to determine amplification success in genome $\mathrm{H}$ of $A$. veigae. The forward $F A D 2 A$ primer spans an insertion upstream of the coding sequence not found in $F A D 2 B$, thereby discriminating the two genomes [19]. Neither primer pair amplified the $F A D 2 H$ gene in the $A$. veigae accessions. Using published peanut $F A D 2$ sequence data, various additional primer combinations were designed and tested. Preliminary sequence data from other Arachis species showed variation in the flanking regions compared to that of cultivated peanut including several indels of varying sizes (unpublished data). Based on these results, a set of primers was designed in conserved regions flanking the FAD2 coding sequence that successfully amplified genome $\mathrm{A}, \mathrm{B}$, and $\mathrm{H}$ species: FAD2F6 and FAD2R6 (5' GTC ACT CTC ATC TGC AAT GAC TAT C $3^{\prime}$ and $5^{\prime}$ ACA TGG CAA ATC CAC ACA CA $3^{\prime}$ ). PCR was performed using $\mathrm{GoTaq}^{\circledR} \mathrm{G} 2$ Green master mix (Promega), and the cycling conditions consisted of 1 cycle of $94{ }^{\circ} \mathrm{C}$ for $5 \mathrm{~min}, 35$ cycles of $94{ }^{\circ} \mathrm{C}$ for $30 \mathrm{~s}, 57^{\circ} \mathrm{C}$ for $30 \mathrm{~s}, 72^{\circ} \mathrm{C}$ for $105 \mathrm{~s}$, and a final extension of $72{ }^{\circ} \mathrm{C}$ for $7 \mathrm{~min}$. Amplicons were sequenced using the PCR primers and two internal primers (5' AAG GGC CAT CCT AGT GTG AG $3^{\prime}$ and $5^{\prime}$ CAT GGT TGG TTT GAC CCT TC $3^{\prime}$ ) to completely cover the coding regions. After trimming each sequence, consensus sequences were assembled using Sequencher, ver. 5.3. By comparing sequences between species and accessions, nonsynonymous SNPs were identified and likely linked to genes responsible for changes in fatty acid composition.

\section{Results and discussion}

Determination and comparison of fatty acid profiles among some selected wild species

The fatty acid composition data from selected wild peanut species are listed in Table 1, and chromatography profiles are shown in Fig. 1. PI 688970 and PI 688983 were originally collected in Brazil by Renato Veiga (Instituto Agronomico) and other colleagues and then donated to PGRCU by Dr. Charles Simpson (Texas A\&M University) in 1993. The original name for this species was A. sylvestris, but it was recently changed to A. veigae [5, https://www.ars-grin.gov]. Seeds from PI 688983 contained a very low percentage of oleic acid (18.27\%) and a very high percentage of linoleic acid (45.7\%) (Table 1). By comparison, PI 688970 contained a high percentage of oleic acid $(55.52 \%)$ and a very low percentage of linoleic acid (8.72\%). The oleic acid level in this mutant was also higher than the oleic acid levels in A. duranensis (PI 468202, 37.98\%) and A. valida (PI 468184, 


\begin{tabular}{|c|c|c|c|c|}
\hline PI number & PI 688970 & PI 688983 & PI 468202 & PI 468184 \\
\hline Species & A. veigae & A. veigae & A. duranensis & A. valida \\
\hline Genome & $\mathrm{H}$ & $\mathrm{H}$ & A & B \\
\hline C16:0 & 3.62 & 7.99 & 10.68 & 11.36 \\
\hline C18:0 & 1.41 & 1.68 & 3.04 & 2.22 \\
\hline C18:1 & 55.52 & 18.27 & 37.98 & 40.15 \\
\hline C18:2 & 8.72 & 45.70 & 39.88 & 33.86 \\
\hline C20:0 & 1.32 & 1.33 & 1.70 & 1.38 \\
\hline C20:1 & 3.59 & 1.54 & 1.22 & 1.57 \\
\hline$C 22: 0$ & 18.15 & 16.99 & 3.71 & 7.16 \\
\hline C22:1 & 1.41 & - & - & - \\
\hline C24:0 & 6.28 & 5.94 & 1.82 & 2.32 \\
\hline $\mathrm{C} 26: 0$ & - & 0.58 & - & - \\
\hline
\end{tabular}

Dash (-) stands for not detectable trace amount of this fatty acid

40.15\%). In addition, accessions of $A$. veigae contained high percentages of very long-chain $(C \geq 22)$ fatty acids. PI 688970 and PI 688983 contained 18.15\% and 16.99\% behenic acid (C22:0) and $6.28 \%$ and $5.94 \%$ lignoceric acid (C24:0), respectively, much higher than PI 468202 (3.71\% and $1.82 \%)$ and PI 468184 (7.16\% and $2.32 \%)$, respectively (Table 1 and Fig. 1). These results are consistent with data collected on other $A$. veigae accessions (not shown) and a previous report [20] where PI 688981 (A. veigae) measured $15.81 \%$ behenic acid and $5.31 \%$ lignoceric acid, higher than most of the other species evaluated in that study. Although the PI 688970 contained only $55.5 \%$ oleic acid (as opposed to $75-80 \%$ which is considered high oleic in cultivated peanut), it also contained a high percentage (25.8\%) of very long-chain fatty acids. This unique fatty acid combination makes this mutant a potential new germplasm resource for oil quality improvement.

\section{Nucleotide and peptide diversity within $A$. veigae and among selected species}

For the 1140-bp coding region of the FAD2 gene and 379-aa peptide of fatty acid desaturase (FAD) enzyme, seven assembled nucleotide sequences and deduced peptide sequences are shown in Figs. 2 and 3, respectively. For this comparison, two nucleotide sequences containing $F A D$ were included from two diploid wild progenitors (A. duranensis, genome A; A. ipaënsis, genome $\mathrm{B}$ ) of the tetraploid cultivated peanut (AABB), two from the $A$. veigae accessions [PI 688983 and PI 688970 (genome $\mathrm{H}$ ], two sequences from subgenomes $A$ and $B$ of cultivated peanut (Tifrunner), and one sequence was from subgenome $B$ of the cultivated peanut F435 (containing a 442A insertion for high oleic acid, designated as subgenome $\mathrm{B}-\mathrm{HO}$ ) [21-23]. For single nucleotide polymorphism (SNP) identification, the sequence from A. duranensis deposited in Genbank was used as a reference, and all other six sequences were compared to it. Number of SNPs identified and amino acid changes are summarized in Table 2. There was little difference between the subgenome A of cultivated peanut Tifrunner and the A genome of $A$. duranensis with only two SNPs identified. This is reasonable because the subgenome A of cultivated peanut most likely originated from the A genome of A. duranensis. One mutation was synonymous (substitution of A907G), while another was nonsynonymous (substitution of G448A) resulting in an amino acid change of D150N (Fig. 3) and $~ 20 \%$ increase in oleic acid [20, 22, 23].

Eleven common SNPs were identified between subgenome $\mathrm{B}-\mathrm{HO}$ of cultivated peanut, genome $\mathrm{B}$ of $A$. ipaënsis and genome A of $A$.duranensis. Additional SNPs were identified for A. ipaënsis (C464T substitution) and for subgenome B-HO (442A insertion), respectively (Fig. 2). There were eight common silent SNPs for subgenome $\mathrm{B}$ of Tifrunner and genome B of A. ipaënsis (Table 2). Three common amino acid changes (V58M, F347V, and $\mathrm{K} 369 \mathrm{Q}$ ) were found for subgenome B of Tifrunner and genome $\mathrm{B}$ of $A$. ipaënsis. There was an additional amino acid change (P155L) for genome $\mathrm{B}$ of $A$. ipaënsis. The previously known and well characterized insertion 442A (a point-nonsense mutation) in subgenome B-HO of the high oleic cultivated F435 resulted in a premature stop codon (Fig. 3) and about 15\% enhancement of oleic acid [20, 22, 23]. A higher number of SNPs were observed from PI 688983 (39) and PI 688970 (41), relative to $A$. duranensis. This indicates that genome $\mathrm{H}$ of $A$. veigae is more diverged from genome $\mathrm{A}$ of $A$. duranensis than genome $\mathrm{B}$ of $A$. ipaënsis in the genome region studied. Within the two accessions of $A$. veigae, 39 SNPs are common with PI 688970 containing two additional SNPs (Fig. 2). There were 10 amino acid changes in PI 688983, but none of these affected the oleic acid content. However, a critical SNP (C37T) exists in PI 688970, which resulted in a premature stop codon $\left(\mathrm{Q} 13^{*}\right)$. This nonsense point mutation likely reduces the catalytic activity of the desaturase enzyme leading to decreased production of linoleic acid and therefore increasing the oleic acid level by $37.25 \%$ (Figs. 1, 2, 3). Interestingly, for PI 688983, there was an amino acid change (A313T) which was located in the histidine box (His box) (Fig. 3). These His boxes were proposed to contribute to fatty acid desaturase activity by [24]; but in this study, the amino acid change in one of the His boxes did not have a significant effect on the level of oleic acid. 

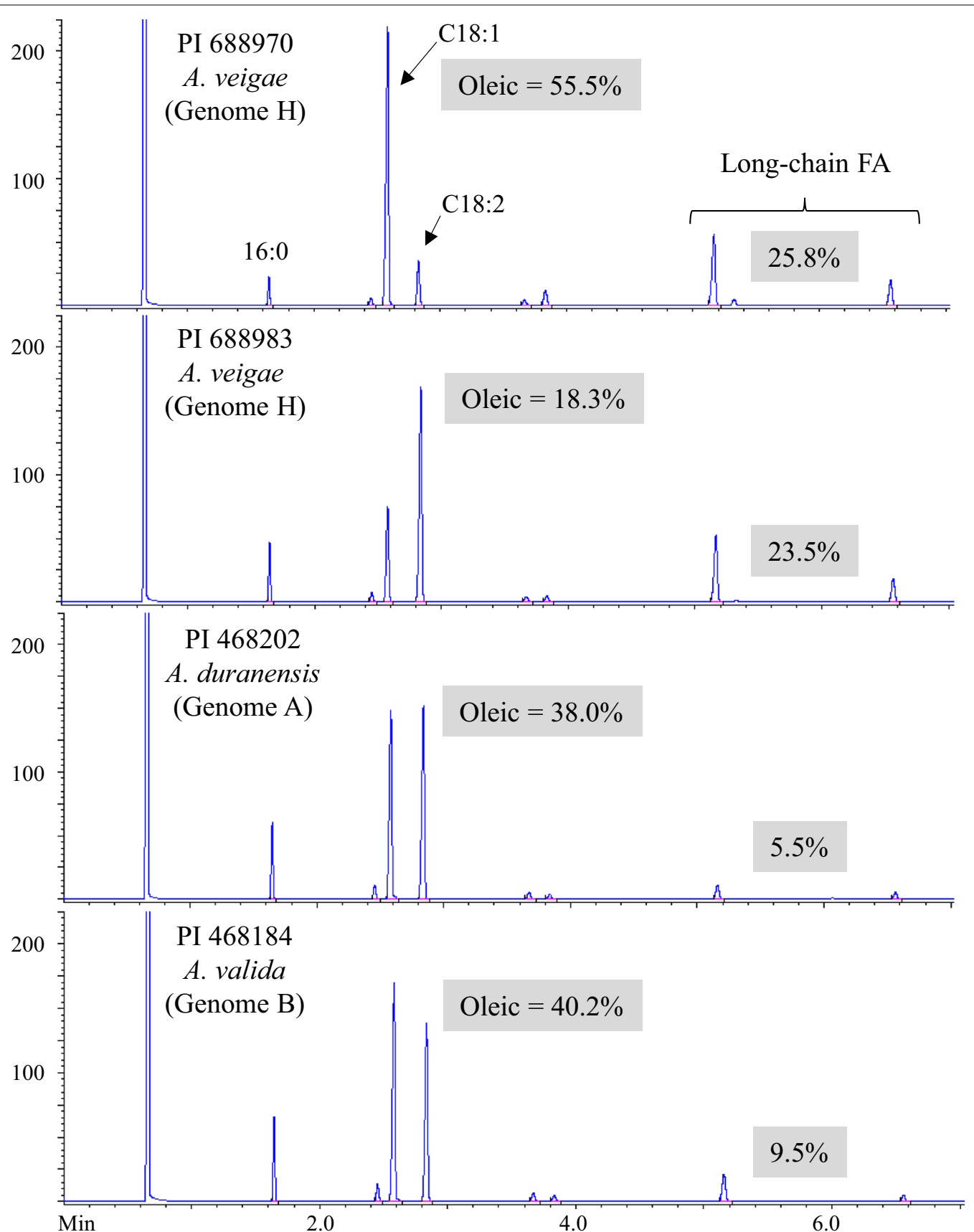

Fig. 1 Comparison of fatty acid profiles among four different wild peanut accessions on chromatograms

Peanut wild relatives are well conserved in germplasm collections with nearly 1000 accessions encompassing 82 species [13], but these wild species are not well exploited for utilization due to crossability barriers and ploidy level differences that lead to sterility in the resulting hybrids. There are many seed quality traits which can be studied, but to date only a few of these (oil, protein, and fatty acid composition) have been evaluated. The accession PI 688970 of $A$. veigae, with $55.5 \%$ oleic acid, is $15-20 \%$ higher than other Arachis species and about 35\% higher than other $A$. veigae accessions; but it is lower than the $75-80 \%$ oleate found in high oleic cultivated peanuts. However, if gene action is additive for fatty acid profiles as reported [25], then the percentage of oleate should 


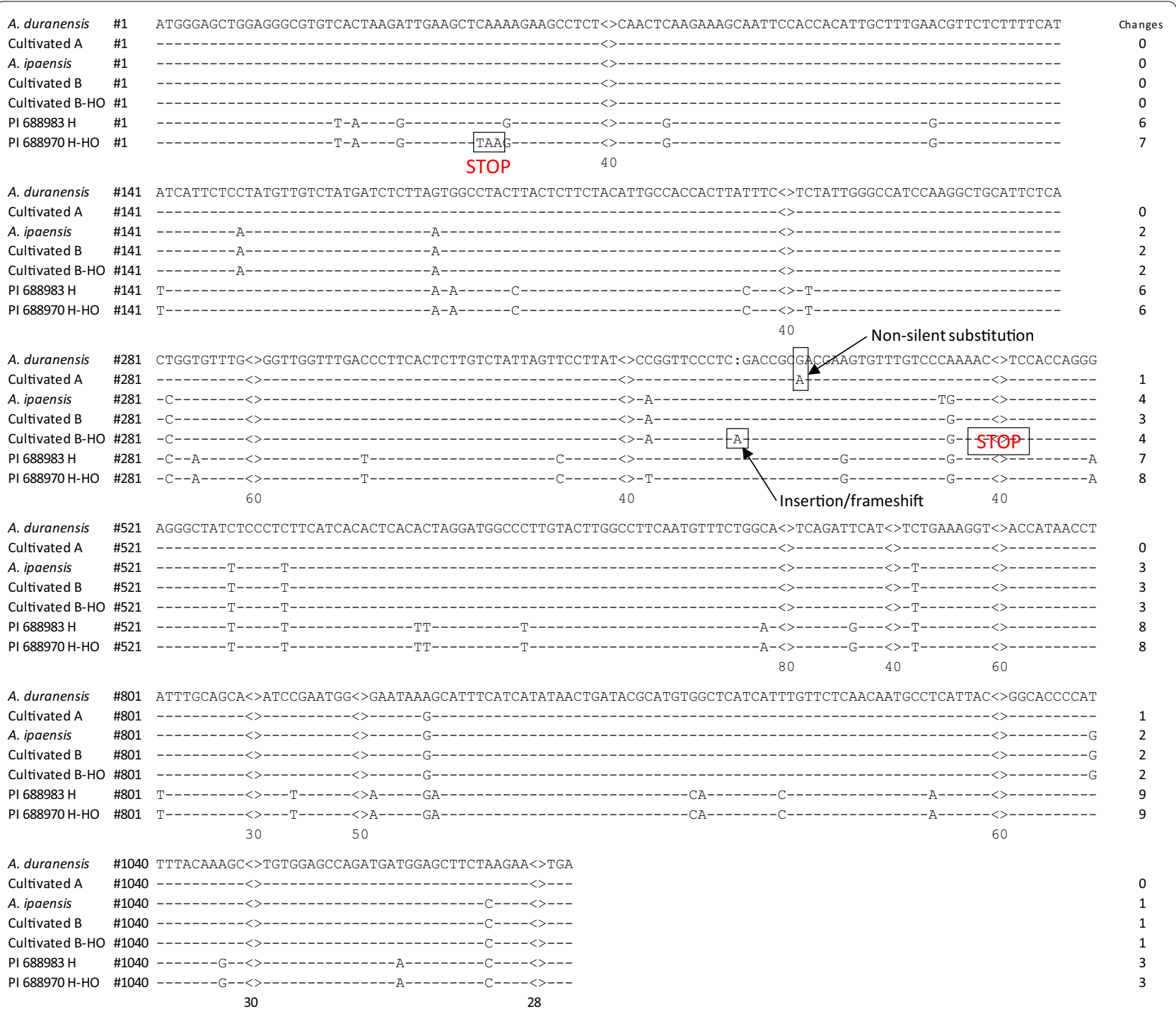

Fig. 2 Comparison of DNA sequences of FAD2 coding region and identification of SNPs among seven genomes from six different lines or accessions. Cultivated A and Cultivated B represent subgenomes A and B from cultivated peanut Tifrunner. Cultivated B-HO represents subgenome B from F435 with high oleic acid trait. " $<>$ " represents omitted stretches with no variation. Black rectangle of A. veigae PI 688970 represents stop codon. There is also a stop codon at position 442 for Cultivated B-HO it is not shown due to the space limitation in the figure. The FAD2 sequences of A. duranesis and A. ipaënsis were downloaded from the public Genbank

increase if the corresponding A. veigae gene (FAD2H$H O)$ can be introgressed into $A$. hypogaea. Since there was no crossability between $A$. veigae and $A$. hypogaea, a 'bridge' species (that can be hybridized with both $A$. veigae and A. hypogaea) should be identified for the introgression of useful traits from A. veigae to A. hypogaea. Possible explanations for the lack of true high oleate mutants similar to the cultivated high oleate identified in the wild species include (a) not enough of the existing germplasm was screened; (b) there are no high oleate mutants in the existing wild species germplasm; (c) the high oleate trait can only be derived from the additive combination of two diploid genomes with an intermediate level $(\sim 50 \%)$ of oleic acid as found in the high oleate cultivated peanut. A fourth explanation is that, despite PI 688970 having a mutation that could result in a high oleate oil, additional genetic changes limit the amount of $\mathrm{C} 18$ fatty acid available for the additional accumulation of oleic acid. Accessions in the section Heteranthae have much higher concentrations of very long-chain fatty 


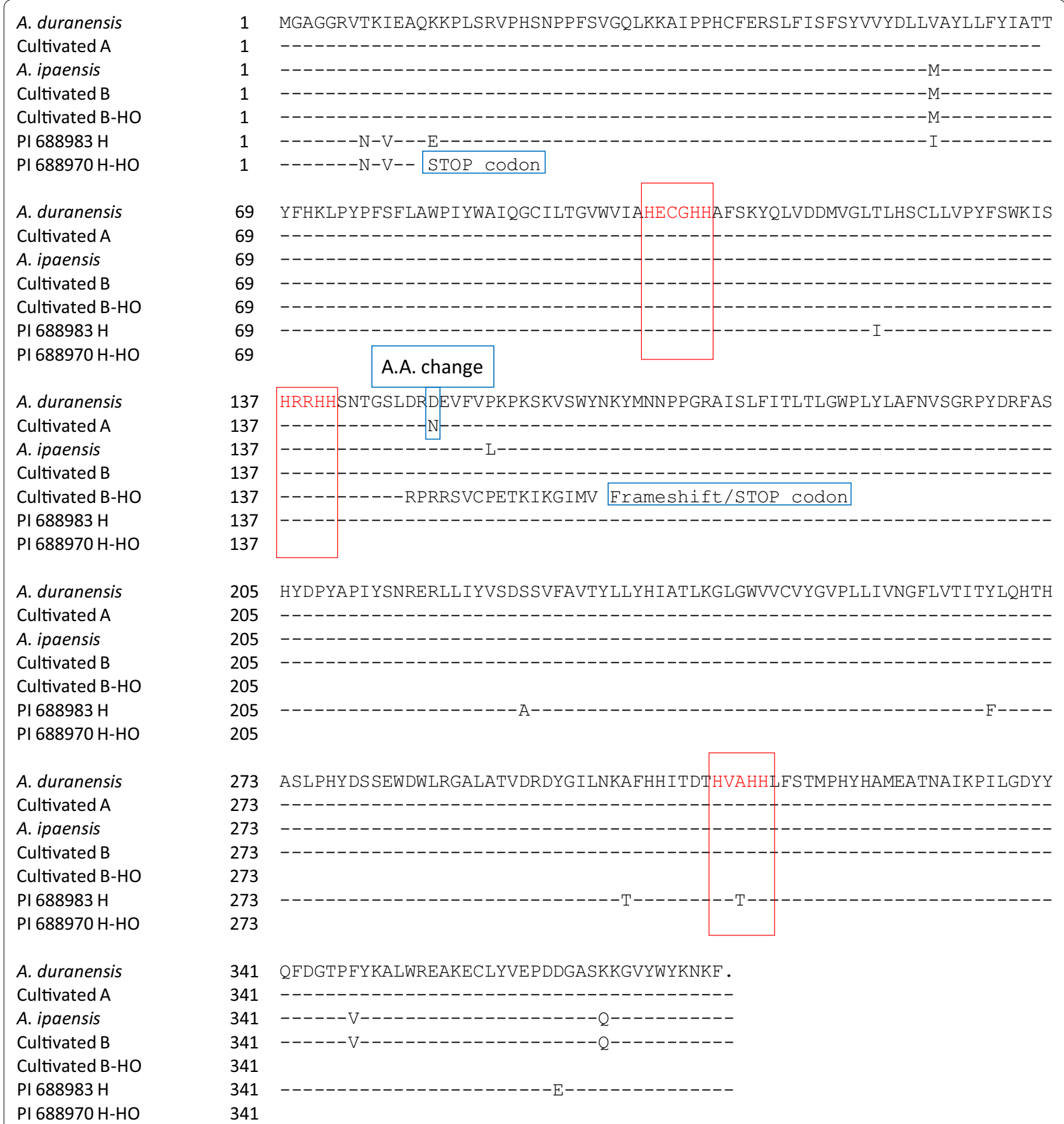

Fig. 3 Comparison of deduced amino acid sequences of fatty acid desaturase (FAD) and identification of amino acid changes. Cultivated A and Cultivated B represent subgenomes A and B from cultivated peanut Tifrunner. Cultivated B-HO represent subgenome B from F435 with high oleic acid trait. Two blue rectangles represent stop codon and frameshift/stop codon. Three red rectangles represent three histidine (His) boxes

acids, thereby reducing the relative concentration of $\mathrm{C} 18$ length fatty acids. Regardless of the reason, this newly identified wild species mutant is unique and may potentially be an important genetic resource for increased oleic acid and very long-chain fatty acids. Further, our results suggest that there is potential for finding other agronomically desirable mutants in the Arachis species germplasm for cultivated peanut improvement. 
Table 2 Comparison of the sequences of FAD2 coding region and amino acid changes among selected cultivated and wild peanut species

\begin{tabular}{|c|c|c|c|c|}
\hline Species & SNP & Silent SNP & Non-silent SNP & $\begin{array}{l}\text { Amino } \\
\text { acid } \\
\text { change }\end{array}$ \\
\hline Tifrunner (cultivated subgenome A) & 2 & 1 & 1 & 1 \\
\hline Tifrunner (cultivated subgenome B) & 11 & 8 & 3 & 3 \\
\hline F435 (cultivated subgenome B-HO) & 12 & Premature stop at 148th codon & & \\
\hline A. ipaënsis (wild genome B) & 12 & 8 & 4 & 4 \\
\hline A. veigae PI 688983 (wild genome H) & 39 & 29 & 10 & 10 \\
\hline A. veigae PI 688970 (wild genome H) & 41 & Premature stop at 13 th codon & & \\
\hline
\end{tabular}

\section{Authors' contributions}

MLW and BT contributed to the design of the research. ST planted wild species and collected seed samples. VT and HTS regrew the mutant plants in the greenhouse. BT conducted DNA sequencing, GC and data analysis. MLW wrote the manuscript and HTS helped to revise the manuscript. All authors read and approved the final manuscript.

\section{Author details}

1 USDA-ARS, Plant Genetic Resources Conservation Unit, University of Georgia, 1109 Experiment Street, Griffin, GA 30223, USA. ${ }^{2}$ Crop and Soil Science Department, University of Georgia, 1109 Experiment Street, Griffin, GA 30223 USA. ${ }^{3}$ Department of Crop and Soil Sciences, North Carolina State University, Raleigh, NC 27695, USA.

\section{Acknowledgements}

The authors gratefully thank Ms. Phiffie Vankus and Sarah Moon for their excellent assistance on seed germination and Drs. Charles Y. Chen and Noelle Anglin for useful suggestions on improving the quality of this manuscript.

\section{Competing interest}

The authors declare that they have no competing interests.

\section{Publisher's Note}

Springer Nature remains neutral with regard to jurisdictional claims in published maps and institutional affiliations.

Received: 6 December 2018 Accepted: 11 February 2019

Published online: 01 March 2019

\section{References}

1. Arya SS, Salve AR, Chauhan S (2016) Peanuts as functional food: a review. J Food Sci Technol 53:31-34

2. Greene SL, Warburton ML (2017) Wading into the gene pool: progress and constraints using wild species. Crop Sci 57:1039-1041

3. Krapovickas A, Gregory WC (1994) Taxonomia del énero Arachis (Leguminosae). Bonplandia 8:1-186

4. Valls JFM, Simpson CE (2005) New species of Arachis (Leguminosae) from Brazil, Paraguay and Bolivia. Bonplandia 14:35-63

5. Santana SH, Valls JFM (2015) Arachis veigae (Fabaceae), the most dispersed wild species of the genus, and yet taxonomically overlooked. Bonplandia 24:139-150

6. Stalker HT, Campbell WV (1983) Resistance of wild species of peanuts to an insect complex. Peanut Sci 10:30-33

7. Stalker HT, Moss JP (1987) Speciation, cytogenetics, and utilization of Arachis species. Adv Agron 41:1-40

8. Burow MD, Simpson CE, Starr JL, Paterson AH (2001) Transmission genetics of chromatin from a synthetic amphidiploid to cultivated peanut (Arachis hypogaea L.): broadening the gene pool of a monophyletic polyploid species. Genetics 159:823-837

9. Simpson CE, Starr UL (2001) Registration of'COAN'peanut. Crop Sci 41:918
10. Gowda MVC, Motagi BN, Naidu GK, Diddimani SB, Sheshagiri R (2002) GPBD 4: a Spanish bunch groundnut genotype resistant to rust and late leaf spot. Int Arachis Newsl 22:29-32

11. Burow MD, Starr JL, Park CH, Simpson CE, Paterson AH (2014) Introgression of homeologous quantitative trait loci (QTLs) for resistance to the root-knot nematode [Meloidogynearenaria (Neal) Chitwood] in an advanced backcross-QTL population of peanut (Arachis hypogaea L.). Mol Breed 34:393-406

12. Leal-Bertioli SCA, Cavalcante U, Gouvea EG, Ballén-Taborda Shirasawa K, Guimãraes PM, Moretzsohn MC (2015) Identification of QTLs for rust resistance in the peanut wild species Arachis magna and the development of KASP markers for marker-assisted selection. G3 Genes Genom Genet 5:1403-1413

13. Stalker HT (2017) Utilizing wild species for peanut improvement. Crop Sci 57:1102-1120

14. Stalker HT, Young CT, Jones TM (1989) A survey of the fatty acids of peanut species. Oleagineaux 44:419-424

15. Grosso NR, Nepote V, Guzmn CA (2000) Chemical composition of some wild peanut species (Arachis L.). J Agric Food Chem 48:806-809

16. Wang ML, Barkley NA, Chinnan M, Stalker HT, Pittman RN (2010) Oil content and fatty acid composition variability in wild peanut species. Plant Genet Resour 8:232-234

17. Tonnis B, Wang ML, Fancher A, Ware T, Tallury SP (2018) Phenotypic variation in seed quality of wild Arachis species. Proc Am Peanut Res Educ Soc 50:30

18. Wang ML, Chen CY, Tonnis B, Barkley NA, Pinnow DL, Pittman RN, Davis J, Holbrook CC, Stalker HT, Pederson GA (2013) Oil, fatty acid, flavonoid, and resveratrol content variability and FAD2A functional SNP genotypes in the U.S. peanut mini-core collection. J Agric Food Chem 61:2875-2882

19. Wang ML, Tonnis B, An YQC, Pinnow D, Tishchenko V, Pederson GA (2015) Newly identified natural high-oleate mutant from Arachis hypogaea L. subsp. hypogaea. Mol Breed 35:186

20. Wang ML, Barkley NA, Chen Z, Pittman RN (2011) FAD2 gene mutations significantly alter fatty acid profiles in cultivated peanut (Arachis hypogaea). Biochem Genet 49:748-759

21. Norden AJ, Borget DW, Knauft DA, Young CT (1987) Variability in oil quality among peanut genotypes in the Florida breeding program. Peanut Sci 4:7-11

22. Jung S, Powell G, Moore K, Abbott A (2000) The high oleate trait in the cultivated peanut [Arachis hypogaea L.]. II. Molecular basis and genetics of the trait. Mol Gen Genet 263:806-811

23. Jung S, Swift D, Sengoku E, Patel M, Teule F, Powell G, Moore K, Abbot A (2000) The high oleate trait in the cultivated peanut [Arachis hypogaea L.]. I. Isolation and characterization of two genes encoding microsomal oleoyl-PC desaturases. Mol Gen Genet 263:796-805

24. Gagné SJ, Reed DW, Gray GR, Covello PS (2009) Structural control of chemoselectivity, stereoselectivity, and substrate specificity in membranebound fatty acid acetylenases and desaturases. Biochem 48:12298-12304

25. Barkley AN, Isleib TG, Wang ML, Pittman RN (2013) Genotypic effect of ahFAD2 on fatty acid profiles in six segeregating peanut (Arachis hypogaea L) populations. BMC Genet 14:62 